\title{
CDK1 and CDC20 overexpression in patients with colorectal cancer are associated with poor prognosis: evidence from integrated bioinformatics analysis
}

\author{
Jianxin Li, Yinchun Wang, Xin Wang and Qingqiang Yang*
}

\begin{abstract}
Background: Colorectal cancer (CRC) is one of the most common malignancies of the digestive system, which causes severe financial burden worldwide. However, the specific mechanisms involved in CRC are still unclear.

Methods: To identify the significant genes and pathways involved in the initiation and progression of CRC, the microarray dataset GSE126092 was downloaded from Gene Expression Omnibus (GEO) database, and then, the data was analyzed to identify differentially expressed genes (DEGs). Subsequently, the Gene Ontology (GO) annotation and Kyoto Encyclopedia of Genes and Genomes (KEGG) pathway analysis were performed on these DEGs using the DAVID database, and the protein-protein interaction (PPI) network was constructed using the STRING database and analyzed using the Cytoscape software. Finally, hub genes were screened, and the survival analysis was performed on these hub genes using the Kaplan-Meier curves in the cBioPortal database.

Results: In total, 937 DEGs were obtained, including 316 upregulated genes and 621 downregulated genes. GO analysis revealed that the DEGs were mostly enriched in terms of nuclear division, organelle fission, cell division, and cell cycle process. KEGG pathway analysis showed that the DEGs were mostly enriched in cell cycle, oocyte meiosis, cytokine-cytokine receptor interaction, and cGMP-PKG signaling pathway. The PPI network comprised 608 nodes and 3100 edges, and 4 significant modules and 10 hub genes with the highest degree were identified using the Cytoscape software. Finally, survival analysis showed that overexpression of CDK1 and CDC20 in patients with CRC were statistically associated with worse overall survival.

Conclusions: This bioinformatics analysis revealed that CDK1 and CDC20 might be candidate targets for diagnosis and treatment of CRC, which provided valuable clues for CRC.

Keywords: Colorectal cancer, Hub genes, Bioinformatics analysis, Cyclin-dependent kinase 1, Cell division cycle 20 homolog
\end{abstract}

\section{Introduction}

Colorectal cancer (CRC) is one of the leading causes of malignancies, which causes severe financial burden worldwide [1,2]. The morbidity of CRC is elevating during the past years, and its age of onset has gradually decreased [3]. The prognosis of patients with CRC is primarily associated with the staging of the primary tumor,

\footnotetext{
*Correspondence: yangqingqiang121@163.com

Department of Gastrointestinal Surgery, The Affiliated Hospital of Southwest Medical University, Luzhou 646000, Sichuan, People's Republic of China
}

patients with CRC at early stages may be cured, and the 5 -year relative survival rate was $90 \%$. However, the prognosis of CRC cases at advanced stages is poor, and recurrence and metastasis are the principal causes of death, despite improvements in surgery-based integrated treatment. Unfortunately, the majority of CRC patients are diagnosed at stages when the cancer cells have metastasized to other organs in the body $[4,5]$. Although numerous efforts have been taken to understand the molecular mechanisms involved in the initiation and progression of $\mathrm{CRC}$, the underlying molecular 
mechanisms and critical genes are still not completely cleared, and the morbidity and mortality of CRC are still rising year by year [6]. Therefore, there is still a need to explore more potential biomarkers for targeted therapy, early diagnosis, and prognosis evaluation of CRC.

The carcinogenesis and progression mechanisms of CRC involve interconnections between environmental and genetic factors; with the rapid development of gene sequencing technology and bioinformatics analysis technology, many researchers have begun to search for the significant differentially expressed genes of CRC through the data mining from online databases, such as the Gene Expression Omnibus (GEO) and The Cancer Genome Atlas (TCGA) [7]. Online databases can obtain expression information of numerous genes simultaneously, and these genes were analyzed to explore the significant genetic alterations associated with the initiation and progression of CRC [8]. Reanalyzing those datasets may find some meaningful information for new research, which provides efficient approaches to identify some potential early diagnostic biomarkers and therapeutic targets for the patients with CRC.

In the present study, the differentially expressed genes between CRC samples and adjacent noncancerous samples were obtained by mining gene expression microarray dataset GSE126092, and the Gene Ontology (GO) annotation and Kyoto Encyclopedia of Genes and Genomes (KEGG) pathway analysis of the DEGs were performed by using the Database for Annotation, Visualization and Integrated Discovery (DAVID) online tool. A protein-protein interaction (PPI) network of DEGs was constructed using the Search Tool for the Retrieval of Interacting Genes (STRING) database and analyzed using the Cytoscape software, and the hub genes were identified. In addition, the survival of patients with hub genes abnormal expression was analyzed using the cBioPortal database.

\section{Materials and methods} Microarray data acquisition

Gene expression profiles of GSE126092 [9] were acquired from the National Center for Biotechnology Information Gene Expression Omnibus database (NCBI GEO, http://www.ncbi.nlm.nih.gov/geo) [10]; GSE126092 consisted of 10 paired CRC and adjacent noncancerous tissues, which was based on the GPL21047 platform (Agilent-074348 Human LncRNA v6 4X180K).

\section{Identification of DEGs}

Before the analysis of DEGs between CRC and adjacent noncancerous tissues, the probe identification numbers of GSE126092 were transformed into gene symbols. When multiple probes corresponded to the same gene, the significant expression value was taken as the gene expression value. After that, the DEGs in the microarray were screened out using limma package in R V3.6.2 (http://www.bioconductor.org/) [11]; the cutoff conditions were set to adjusted $P$ value $<0.05$ and absolute value of $\log$ fold change $\left(\log _{2} \mathrm{FC}\right) \geq 2$.

\section{Gene ontology and pathway enrichment analysis}

To further clarify the potential functional annotation and pathway enrichment associated with the DEGs, Gene Ontology (GO) analysis including biological process, cellular component, and molecular function and Kyoto Encyclopedia of Genes and Genomes (KEGG) pathway analysis were completed with the Database for Annotation, Visualization and Integrated Discovery (DAVID, http://david.abcc.ncifcrf.gov/) (version 6.8) [12, 13]. DAVID is an online tool with gene annotation, visualization, gene ID conversion, and integrated discovery function, and thus can provide the biological significance of genes. Only terms with $P$ values of $<0.05$ and the number of enriched genes $\geq 2$ were considered statistically significant.

\section{Integration of protein-protein interaction network and module analysis}

The protein-protein interaction (PPI) network of DEGs was constructed using the online database Search Tool for the Retrieval of Interacting Genes (STRING, http:// string-db.org) (version 11.0) [14], and a confidence score of $\geq 0.4$ was set as the threshold. The protein nodes which have no interaction with other proteins were removed. Furthermore, to screen the significant modules and hub genes from the PPI network, the PPI network was analyzed using the Cytoscape software (http://www. cytoscape.org/) (version 3.7.2) [15]. The MCODE plugin was used to select significant clustering modules with the criteria of MCODE score $>3$ and the number of nodes > 10, and GO and KEGG analyses of the genes in these modules were performed using DAVID. Subsequently, the CytoHubba plug-in was used to screen the PPI network, and the top 10 genes, which were the hub genes in the $\mathrm{CRC}$, were identified using the degree algorithm.

\section{Survival analysis and validation of gene expression}

In order to identify the potential prognostic role of these hub genes, the survival analysis of hub genes was performed using the Kaplan-Meier curves in the cBioPortal database (http://www.cbioportal.org) [16], and log rank test $P$ value $<0.05$ being the threshold of statistical significance. Then, the UCSC Cancer Genomics Browser (https://genome-cancer.ucsc.edu/) was used for hierarchical clustering of these hub genes [17]. Finally, a comparison of expression of these genes in multiple 
databases was analyzed using the online database Oncomine (http://www.oncomine.com) [18].

\section{Results}

\section{Identification of DEGs in CRC}

In the present study, the row data of GSE126092 dataset, including 10 paired CRC and adjacent noncancerous tissues, was downloaded from the GEO database. The median value of each sample was normalized (Fig. 1a). A total of 937 genes were identified, including 316 upregulated genes and 621 downregulated genes. The volcano plot of DEGs was presented in Fig. 1b, and the expression heat map of the top 50 upregulated and the top 50 downregulated DEGs were presented in Fig. 1c.

\section{GO and KEGG enrichment analyses of DEGs}

To further explore the biological function of the DEGs, the DAVID database was used to perform GO and
KEGG enrichment analysis. As shown in Table 1, in the biological process (BP) group, upregulated DEGs were primarily enriched in nuclear division, organelle fission, cell division, and cell cycle process, while downregulated DEGs were primarily enriched in muscle system process, regulation of system process, and system process. In the cellular component $(\mathrm{CC})$ group, upregulated DEGs were primarily enriched in chromosome and chromosome centromeric region, while downregulated DEGs were primarily enriched in neuron projection and neuron part. In the molecular function (MF) group, upregulated genes were primarily enriched in microtubule motor activity, chemokine activity, and motor activity, while downregulated DEGs were primarily enriched in ion channel activity, channel activity, and passive transmembrane transporter activity. Moreover, KEGG pathway analysis results showed that the upregulated DEGs were significantly enriched in cell cycle, oocyte meiosis, and cytokine-cytokine receptor interaction,

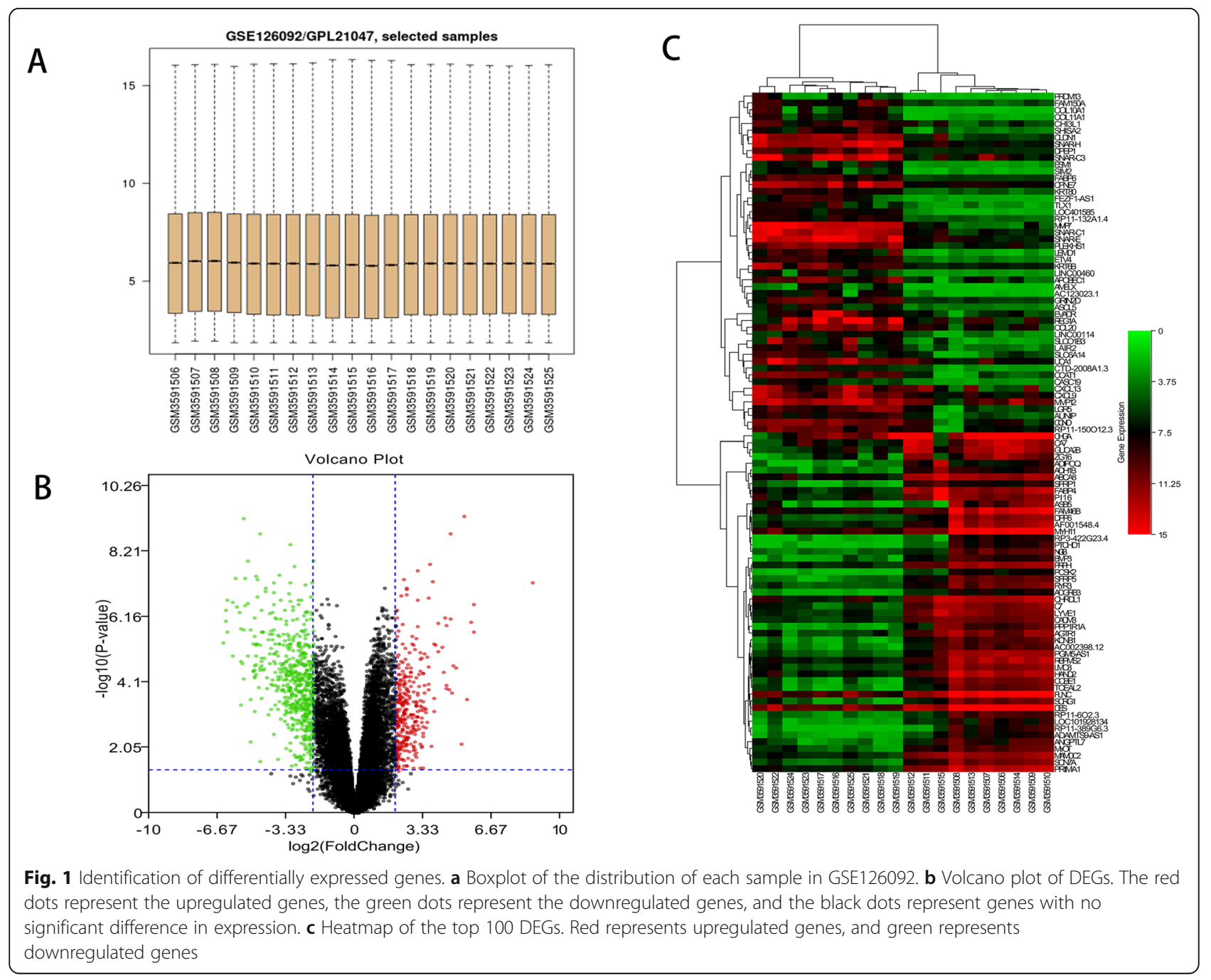


Table 1 Gene Ontology and KEGG pathway analysis of DEGs in colorectal cancer

\begin{tabular}{|c|c|c|c|c|}
\hline Expression & Category & Term & Count & $P$ value \\
\hline \multirow[t]{20}{*}{ Upregulated } & GOTERM_BP_FAT & GO:0000280 nuclear division & 40 & $1.34 \mathrm{E}-18$ \\
\hline & GOTERM_BP_FAT & GO:0048285 organelle fission & 40 & $1.23 \mathrm{E}-17$ \\
\hline & GOTERM_BP_FAT & GO:0051301 cell division & 37 & $2.68 \mathrm{E}-16$ \\
\hline & GOTERM_BP_FAT & GO:0022402 cell cycle process & 56 & 4.22E-16 \\
\hline & GOTERM_BP_FAT & GO:0007049 cell cycle & 61 & $3.43 \mathrm{E}-15$ \\
\hline & GOTERM_CC_FAT & GO:0005694 chromosome & 41 & 1.95E-11 \\
\hline & GOTERM_CC_FAT & GO:0000775 chromosome, centromeric region & 18 & $4.41 \mathrm{E}-10$ \\
\hline & GOTERM_CC_FAT & GO:0005819 spindle & 22 & $4.71 \mathrm{E}-10$ \\
\hline & GOTERM_CC_FAT & GO:0044427 chromosomal part & 36 & $5.61 \mathrm{E}-10$ \\
\hline & GOTERM_CC_FAT & GO:0098687 chromosomal region & 22 & $5.14 \mathrm{E}-09$ \\
\hline & GOTERM_MF_FAT & GO:0003777 microtubule motor activity & 10 & 4.37E-07 \\
\hline & GOTERM_MF_FAT & GO:0008009 chemokine activity & 7 & $2.38 \mathrm{E}-05$ \\
\hline & GOTERM_MF_FAT & GO:0003774 motor activity & 10 & 4.01E-05 \\
\hline & GOTERM_MF_FAT & GO:0045236 CXCR chemokine receptor binding & 5 & 4.08E-05 \\
\hline & GOTERM_MF_FAT & GO:0005125 cytokine activity & 12 & $7.12 \mathrm{E}-05$ \\
\hline & KEGG_PATHWAY & hsa04110:Cell cycle & 15 & $9.41 \mathrm{E}-10$ \\
\hline & KEGG_PATHWAY & hsa04114:Oocyte meiosis & 10 & $1.81 \mathrm{E}-05$ \\
\hline & KEGG_PATHWAY & hsa04914:Progesterone-mediated oocyte maturation & 9 & 2.17E-05 \\
\hline & KEGG_PATHWAY & hsa04060:Cytokine-cytokine receptor interaction & 13 & $1.05 E-04$ \\
\hline & KEGG_PATHWAY & hsa05322:Systemic lupus erythematosus & 8 & 0.002278 \\
\hline \multirow[t]{20}{*}{ Downregulated } & GOTERM_BP_FAT & GO:0003012 muscle system process & 43 & $1.93 \mathrm{E}-15$ \\
\hline & GOTERM_BP_FAT & GO:0044057 regulation of system process & 45 & $1.99 E-13$ \\
\hline & GOTERM_BP_FAT & GO:0006936 muscle contraction & 33 & $3.28 \mathrm{E}-11$ \\
\hline & GOTERM_BP_FAT & GO:0003008 system process & 96 & $1.06 \mathrm{E}-10$ \\
\hline & GOTERM_BP_FAT & GO:0007399 nervous system development & 100 & $2.43 E-10$ \\
\hline & GOTERM_CC_FAT & GO:0043005 neuron projection & 60 & 1.19E-10 \\
\hline & GOTERM_CC_FAT & GO:0042383 sarcolemma & 19 & $5.73 \mathrm{E}-10$ \\
\hline & GOTERM_CC_FAT & GO:0097458 neuron part & 67 & 4.47E-08 \\
\hline & GOTERM_CC_FAT & GO:0044449 contractile fiber part & 22 & 4.57E-08 \\
\hline & GOTERM_CC_FAT & GO:0030425 dendrite & 34 & 7.24E-08 \\
\hline & GOTERM_MF_FAT & GO:0005216 ion channel activity & 32 & 5.05E-08 \\
\hline & GOTERM_MF_FAT & GO:0015267 channel activity & 34 & $5.49 \mathrm{E}-08$ \\
\hline & GOTERM_MF_FAT & GO:0022803 passive transmembrane transporter activity & 34 & 5.77E-08 \\
\hline & GOTERM_MF_FAT & GO:0022838 substrate-specific channel activity & 32 & 1.16E-07 \\
\hline & GOTERM_MF_FAT & GO:0005539 glycosaminoglycan binding & 20 & $6.73 \mathrm{E}-07$ \\
\hline & KEGG_PATHWAY & hsa04022:CGMP-PKG signaling pathway & 13 & 8.87E-04 \\
\hline & KEGG_PATHWAY & hsa05410:Hypertrophic cardiomyopathy (HCM) & 9 & $9.58 \mathrm{E}-04$ \\
\hline & KEGG_PATHWAY & hsa05414:Dilated cardiomyopathy & 9 & 0.001557 \\
\hline & KEGG_PATHWAY & hsa04920:Adipocytokine signaling pathway & 8 & 0.002302 \\
\hline & KEGG_PATHWAY & hsa04261:Adrenergic signaling in cardiomyocytes & 11 & 0.00334 \\
\hline
\end{tabular}

The top five terms of GO and KEGG analysis were selected according to $P$ value. $G O$ Gene Ontology, $B P$ biological process, $C C$ cellular component, $M F$ molecular function, KEGG Kyoto Encyclopedia of Genes and Genomes 
while downregulated DEGs were significantly enriched in cGMP-PKG signaling pathway, hypertrophic cardiomyopathy, and adipocytokine signaling pathway.

\section{Integration of protein-protein interaction network and module analysis}

The STRING online database was used to construct a PPI network consisting of 608 nodes and 3100 edges, and this network was then analyzed using the Cytoscape software. Four significant clustering modules were selected using the MCODE plug-in, and the functional annotation of the DEGs involved in these modules was analyzed (Fig. 2). Clustering module 1 consisting of 50 nodes and 1129 edges, the genes in module 1 were primarily associated with mitotic nuclear division, cell division, and P53 signaling pathway. Clustering module 2 consisting of 13 nodes and 78 edges, the genes in module 2 were primarily associated with chemokinemediated signaling pathway, G protein-coupled receptor pathway, and TNF signaling pathway. Clustering module 3 consisting of 23 nodes and 62 edges, the genes in module 3 were primarily associated with protein ubiquitination, signal transduction, and cytokinecytokine receptor interaction. Clustering module 4 consisting of 27 nodes and 48 edges, the genes in module 4 were primarily associated with Wnt signaling pathway, heterotypic cell-cell adhesion, and ECM-receptor interaction. Furthermore, the top 10 hub genes (CDK1, CDC20, AURKA, PLK1, AURKB, CDC6, KIF11, CCNA2, CENPE, and MKI67) were screened using the degree algorithm of the CytoHubba plug-in; the full name and function of these hub genes are listed in Table 2. Among these hub genes, CDK1 and CDC20 showed the highest degree.

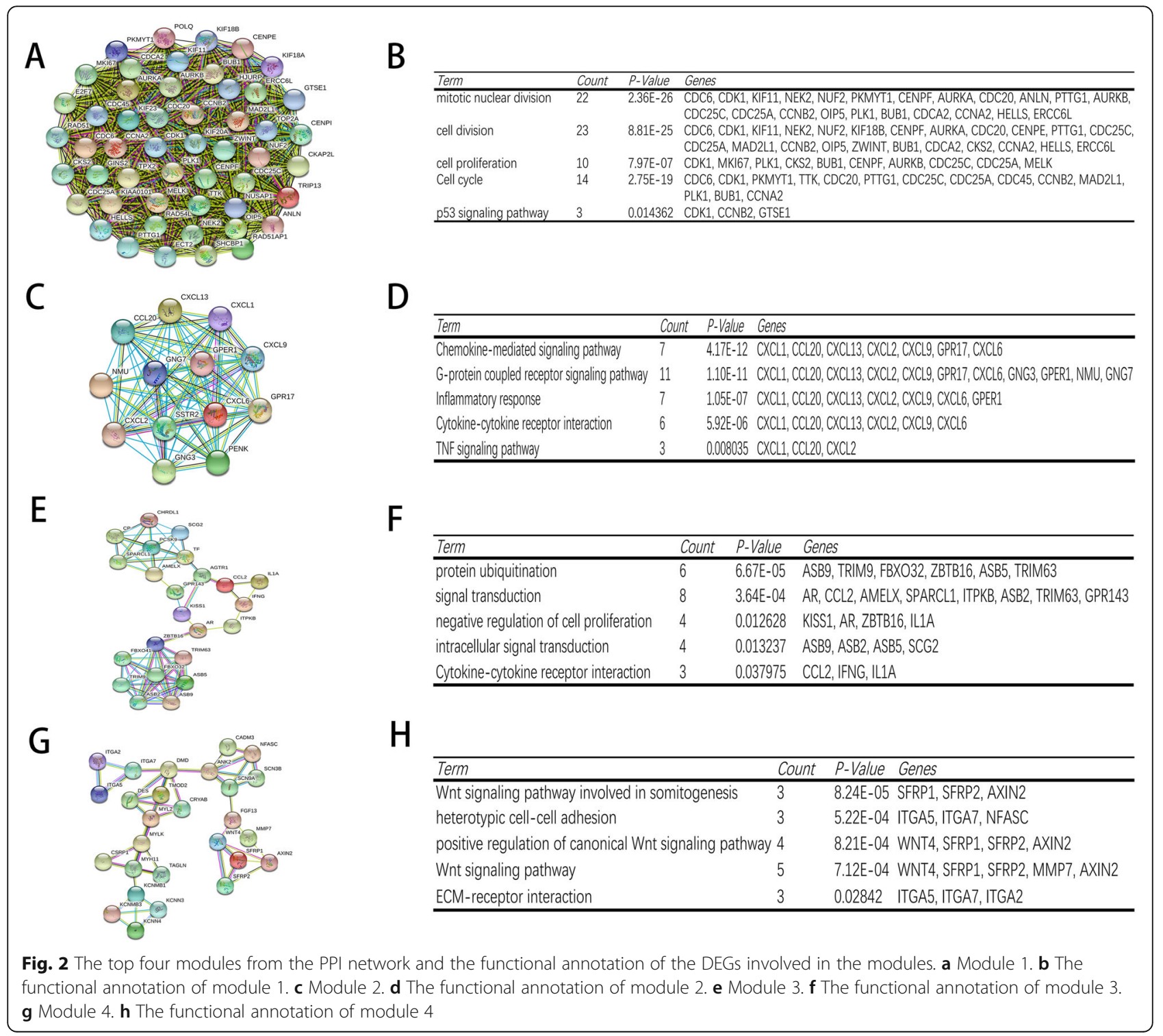


Table 2 Functional roles of 10 hub genes

\begin{tabular}{|c|c|c|c|}
\hline No. & $\begin{array}{l}\text { Gene } \\
\text { symbol }\end{array}$ & Full name & Function \\
\hline 1 & CDK1 & Cyclin-dependent kinase 1 & $\begin{array}{l}\text { Plays a key role in the control of the eukaryotic cell cycle by modulating the centrosome cycle as well } \\
\text { as mitotic onset }\end{array}$ \\
\hline 2 & CDC20 & $\begin{array}{l}\text { Cell division cycle protein } \\
20 \text { homolog }\end{array}$ & $\begin{array}{l}\text { Required for full ubiquitin ligase activity of the anaphase promoting complex/cyclosome and may } \\
\text { confer substrate specificity upon the complex }\end{array}$ \\
\hline 3 & AURKA & Aurora kinase A & Mitotic serine/threonine kinase that contributes to the regulation of cell cycle progression. \\
\hline 4 & PLK1 & Polo-like kinase 1 & $\begin{array}{l}\text { Serine/threonine-protein kinase that performs several important functions throughout M phase of the } \\
\text { cell cycle }\end{array}$ \\
\hline 5 & AURKB & Aurora kinase B & $\begin{array}{l}\text { Serine/threonine-protein kinase component of the chromosomal passenger complex (CPC), a complex } \\
\text { that acts as a key regulator of mitosis }\end{array}$ \\
\hline 6 & CDC6 & $\begin{array}{l}\text { Cell division control protein } \\
6 \text { homolog }\end{array}$ & $\begin{array}{l}\text { Involved in the initiation of DNA replication. Also participates in checkpoint controls that ensure DNA } \\
\text { replication is completed before mitosis is initiated }\end{array}$ \\
\hline 7 & KIF11 & Kinesin family member 11 & $\begin{array}{l}\text { Motor protein required for establishing a bipolar spindle during mitosis. Required in nonmitotic cells for } \\
\text { transport of secretory proteins from the Golgi complex to the cell surface }\end{array}$ \\
\hline 8 & CCNA2 & Cyclin A2 & Cyclin which controls both the G1/S and the G2/M transition phases of the cell cycle \\
\hline 9 & CENPE & $\begin{array}{l}\text { Centromere-associated } \\
\text { protein } \mathrm{E}\end{array}$ & $\begin{array}{l}\text { Microtubule plus-end-directed kinetochore motor which plays an important role in chromosome con- } \\
\text { gression, microtubule-kinetochore conjugation, and spindle assembly checkpoint activation }\end{array}$ \\
\hline 10 & MKI67 & $\begin{array}{l}\text { Marker of proliferation Ki- } \\
67\end{array}$ & $\begin{array}{l}\text { Required to maintain individual mitotic chromosomes dispersed in the cytoplasm following nuclear } \\
\text { envelope disassembly }\end{array}$ \\
\hline
\end{tabular}

\section{Survival analysis and validation of gene expression}

To investigate the expression of hub genes in CRC, a hierarchical clustering analysis was performed using the UCSC Cancer Genomics Browser, revealing that these 10 hub genes were highly expressed in most CRC samples (Fig. 3a). Subsequently, the survival analysis of these 10 hub genes was performed using the Kaplan-Meier curves in the cBioPortal database. The results revealed that patients with CDK1 and CDC20 alteration showed worse overall survival (Fig. $3 \mathrm{~b}, \mathrm{~d}$ ), and the expression levels of CDK1 and CDC20 genes were differentially expressed in CRC samples and noncancerous samples (Fig. 3c, e). However, the differential expression of AURKA, PLK1, AURKB, CDC6, KIF11, CCNA2, CENPE, and MKI67 were not significantly associated with the survival of CRC patients $(P>0.05)$. Oncomine analysis revealed that $\mathrm{CDK} 1$ and $\mathrm{CDC} 20$ expression were obviously elevated in most cancers, especially in breast cancer, colorectal cancer, and lung cancer (Fig. 4a, b). In addition, CDK1 and CDC20 were significantly overexpressed in CRC in multiple CRC datasets (Fig. 4c, d). These findings indicated that CDK1 and CDC20 might be candidate targets for diagnosis and treatment of CRC.

\section{Discussion}

Colorectal cancer (CRC) is the third most common malignant tumor in the world, and the morbidity of CRC has gradually increased over the past decades [1]. The initiation and progression of CRC are involved; although numerous studies have been conducted to reveal the molecular mechanisms of CRC initiation and progression over the past decade, the morbidity of CRC is still very high. Therefore, there is a further need to explore the molecular mechanisms of CRC for early diagnosis and treatment of the disease. The development of microarray technology and gene sequencing technology has helped to understand the pathogenesis of colorectal cancer at the molecular level.

Therefore, the present study aims to investigate the causes of CRC initiation and progression, and gene expression data of 10 paired CRC and adjacent noncancerous tissues were obtained from the GEO database. A total of 937 DEGs, including 316 upregulated genes and 621 downregulated genes, were identified by bioinformatics analysis. In order to further explore the biological function of these DEGs, functional annotation analysis of these DEGs was performed by using the DAVID database. GO analysis revealed that the upregulated DEGs were mostly associated with nuclear division, organelle fission, cell division, and cell cycle process, while downregulated DEGs were primarily associated with the muscle system process, regulation of system process, and system process. KEGG pathway analysis revealed that the upregulated DEGs were mostly associated with cell cycle and cytokine-cytokine receptor interaction, while downregulated DEGs were significantly enriched in the CGMP-PKG signaling pathway. These results above showed that the upregulated DEGs might influence CRC initiation and progression by regulating cell division and cell cycle process, and the downregulated DEGs may be linked to the initiation and progression of CRC by regulating signal transduction pathways. Previous studies indicated that dysregulation of the mechanisms that control cell proliferation, differentiation, and 


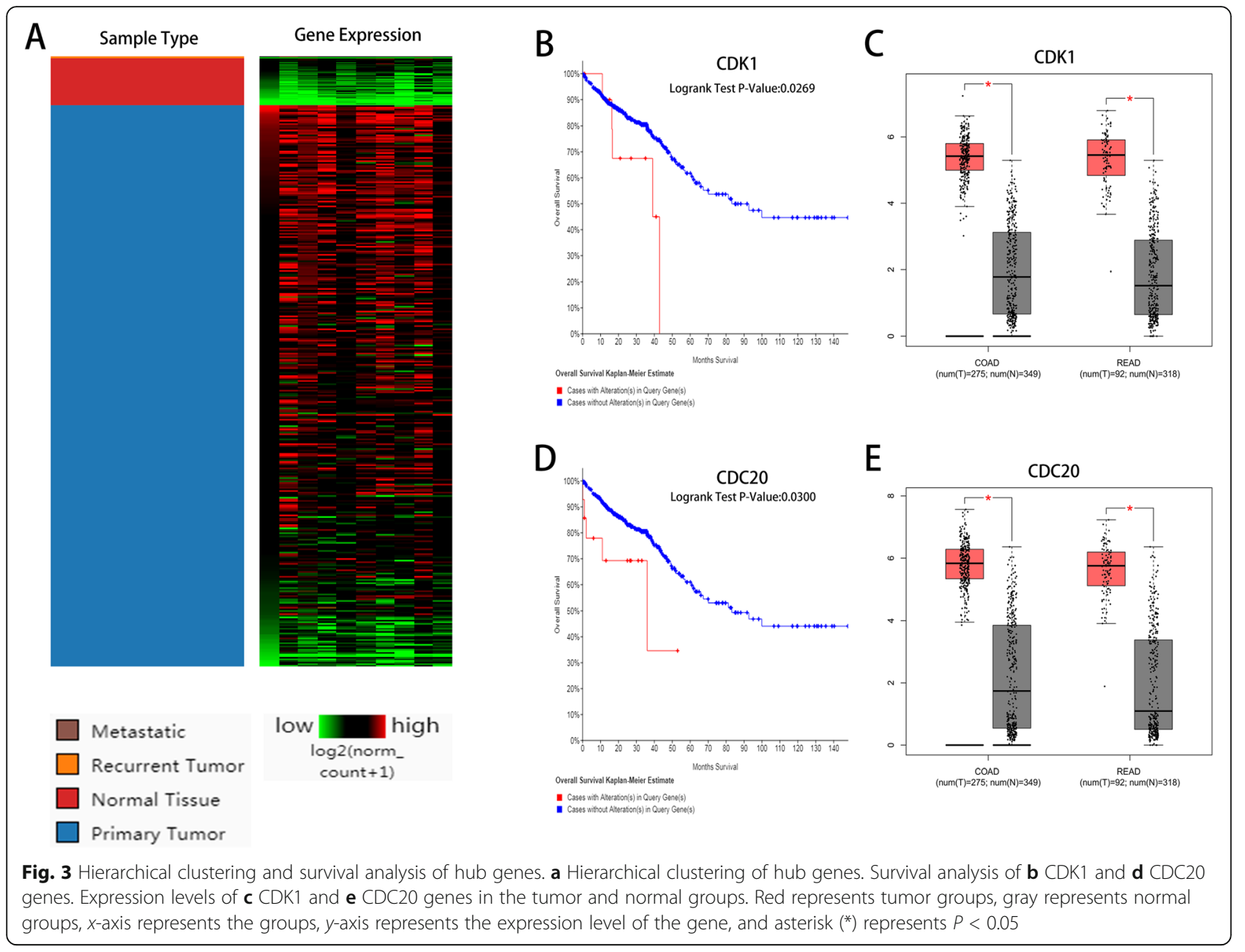

apoptosis has long been considered as a mechanism for cancer cells to gain a growth advantage over noncancerous cells [19]. Cytokines are involved in regulating immune response, stimulating cell activation, proliferation, and differentiation, and many studies have pointed out that the dysregulation of cytokine, such as TNF $\alpha$, IL- $1 \beta$, and IL-22, was associated with the pathological process of CRC [20, 21]. Recent researches also pointed out that the cGMP/PKG cascade is recognized as an endogenous apoptotic pathway in numerous cancer types, including CRC [22]. Browning et al. [23] indicated that PKG1 could block tumor growth and angiogenesis in xenografts by reducing the production of VEGF by tumor cells, and cGMP-dependent protein kinase may be potential targets for colon cancer prevention and treatment.

We constructed a PPI network with all DEGs, and the module analysis of the PPI networks suggested that the carcinogenesis and progression of CRC were associated with cell cycle, P53 signaling pathway, chemokinemediated signaling pathway, TNF signaling pathway, protein ubiquitination, Wnt signaling pathway, and
ECM-receptor interaction. P53 functions as one of the most critical tumor suppressor gene, which is the most common mutational gene in human cancer [24]. Cooks et al. [25] reported that the dysregulation of the P53 signaling pathway might exacerbate the inflammatory response and protects the mutant cells from the clearance of the immune system by enhancing the activity of NF$\mathrm{kB}$, which promoted the carcinogenesis and progression of chronic inflammation and inflammation-associated CRC. Schulz-Heddergott et al. [26] revealed that the decrease of p53 mutation level could inhibit the carcinogenesis and progression of CRC. Mitchell et al. [27] indicated that the abnormal expression of chemokines or chemokine receptors in CRC might contribute to identify the molecular characteristics of tumors and predict the clinical prognosis of patients; several chemokines and chemokine receptors have been shown to promote $\mathrm{CRC}$ metastasis. Aberrations in the ubiquitin system also have been reported to be associated with various diseases, including cancer, for example, with elevated expression of Cathepsin D and Ubiquitin C- 


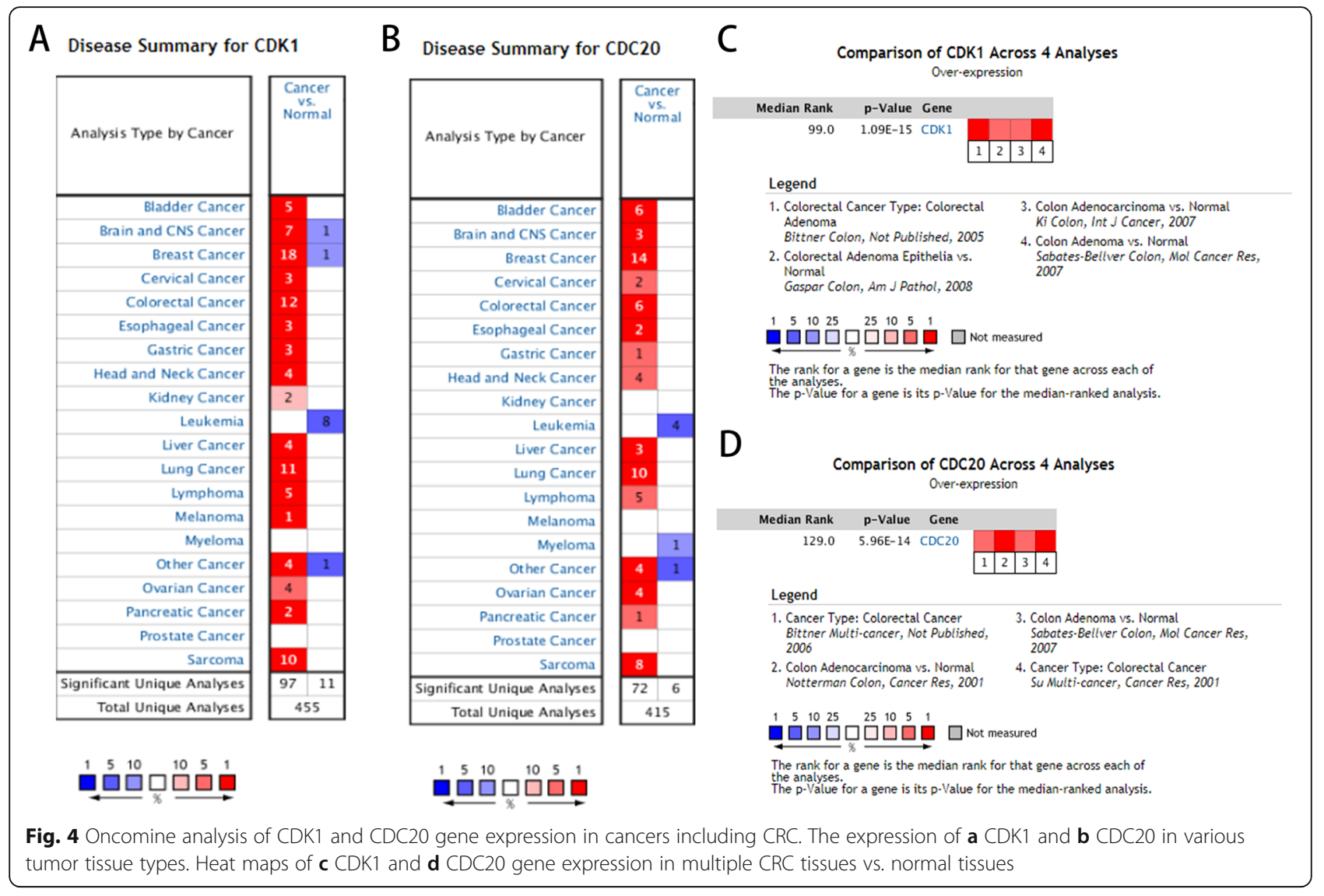

terminal hydrolase- $\mathrm{L} 1$ in tumor cells, the incidence of lymph node metastasis in CRC also has increased [28]. Previous studies have reported that the Wnt signaling pathway was altered in $93 \%$ of all tumors, approximately $90 \%$ of CRC patients with the dysregulation of the Wnt signaling pathway [29]. Moreover, de Sousa e Melo and Vermeulen [30] pointed out that the aberrant regulation of Wnt signaling is associated with chemotherapeutic resistance in CRC patients. ECM-receptor interaction pathway also plays a critical role in the process of proliferation, differentiation, and metastasis of cancer cells; Rahbari et al. [31] reported that the ECM could promote the metastasis of CRC by inducing epithelial-mesenchymal transition (EMT) in tumor cells.

After further analysis of the PPI network, 10 hub genes (CDK1, CDC20, AURKA, PLK1, AURKB, CDC6, KIF11, CCNA2, CENPE, and MKI67) with the highest degrees of interaction were screened, and the expression levels of all these genes showed constant upregulation compared to the gene expression levels of the normal mucosa. Furthermore, results of survival analysis showed that the patients with high gene expression levels of CDK1 and CDC20 showed a worse prognosis. These results suggested that $\mathrm{CDK} 1$ and $\mathrm{CDC} 20$ are involved in the recurrence and metastasis of $\mathrm{CRC}$, and these two genes may be novel targets for early diagnosis and therapy of CRC.

As a member of the cyclin-dependent kinases (CDKs) family, cyclin-dependent kinase 1 (CDK1) acts as a vital driver of cell cycle transition [32]. Over the past decade, a large number of researches have shown that dysregulation of CDK1 not only causes rapid tumor growth, but also leads to the spontaneous proliferation of cancer cells [33]. Previous studies have shown that CDK1 is involved in the progression of multiple types of cancer, including colorectal cancer, liver cancer, and lung cancer. Furthermore, the upregulation of CDK1 is associated with reduced survival time for these diseases [34-36]. Xue et al. [37] reported that the activity of CDK1 is highly elevated in CRC tissues compared to noncancerous tissues, and it predicts distant metastasis risk in CRC; CDK1 can promote the progression of CRC through phosphorylation of JAK1 to activate the JAK/ STAT3 signaling pathway. Bury et al. [38] indicated that high gene expression levels of CDK1 stimulated the proliferation and migration of colorectal cancer cells, and inhibition of CDK1 activity by using inhibitors can inhibit the proliferation of colorectal cancer cells in vitro and in vivo. In addition, Zhang et al. [39] indicated that frequent overexpression of CDK1 in human CRCs is 
associated with the therapeutic target, and the therapeutic resistance of BRAF mutant human CRC can be suppressed by targeting CDK1.

Cell division cycle 20 homolog (CDC20), a homolog of the cell division cycle 20 protein in saccharomyces cerevisiae, has long been recognized as one of the significant regulatory components of the cell cycle and plays a significant role in carcinogenesis and progression of various malignancies [40]. Kidokoro et al. [41] reported that CDC20 is highly expressed in the vast majority of malignancies, including CRC, and the expression level of $\mathrm{CDC} 20$ is negatively regulated by $\mathrm{p} 53$, with the silencing of CDC20 significantly inhibiting cell growth in vitro. In addition, elevated $\mathrm{CDC} 20$ levels have been found to be associated with clinical stage, pathologic differentiation, and TNM stage in CRC, and patients with overexpression of $\mathrm{CDC} 20$ had a shorter overall survival than those with low expression of CDC20 [42]. Moreover, Hadjihannas et al. [43] reported that Wnt/ $\beta$-catenin signaling pathway, which acts as a critical role in embryonic development, stem cell maintenance, and carcinogenesis, is regulated by $\mathrm{CDC} 20$ via controlling the levels of conduction protein during the cell cycle, and CDC20 knockdown inhibited the proliferation of CRC cells. These results suggested that high expression of $\mathrm{CDC} 20$ was an independent prognostic factor, and $\mathrm{CDC} 20$ serves as a potential prognostic biomarker for patients with CRC.

Consistent with our studies, previous studies also indicated that the abnormal expression of other hub genes in our study were associated with the initiation and progression of CRC. Aurora kinases A (AURKA) and Aurora kinases $B$ (AURKB) were members of the Aurora kinase family, which serves as critical regulators of mammalian mitosis, and have been described to be associated with chromosomal instability, aggressive growth, and worse prognosis in various malignancies including CRC [44]. Goos et al. [45] revealed that AURKA overexpression is involved in the process of liver metastasis after colorectal cancer resection, and AURKA inhibitors could be developed as therapeutic agents for CRC. In addition, studies also have been reported that high gene expression levels of AURKB were significantly associated with decreased overall survival in patients with CRC [44]. Polo-like kinase 1 (PLK1) was one of the most significant member of the Polo-like kinase family and plays an important role in cell division and checkpoint regulation during mitosis; with the depleting of PLK1, the proliferation of WT p53-expressing CRC cells was inhibited [46]. Cell division control protein 6 homolog (CDC6), a central regulator of DNA replication and cell proliferation, has been reported to act as a potential oncogenic target in multiple tumors. CDC6 knockdown inhibited CRC cell malignant behaviors (e.g., growth, DNA synthesis, EMT) and oxaliplatin resistance in vitro [47]. Kinesin family member 11 (KIF11) belongs to the kinesin-like protein family, which encodes the motor protein. Imai et al. [48] reported that overexpression of KIF11 is an early event in the pathogenesis of CRC. However, the effect of KIF11 on the prognosis of patients with CRC remains unclear. There is also increasing evidence that cyclin A2 (CCNA2) could be a novel biomarker for diagnosis and therapy for CRC. Gan et al. [49] reported that CCNA2 was overexpressed in CRC tissues and cell lines, and CCNA2 knockdown significantly inhibited the proliferation of CRC cells by inhibiting cell cycle progression and inducing apoptosis. Centrosome-associated protein $\mathrm{E}$ (CENPE) is a kinesin-like motor protein, which accumulates in the G2 phase of the cell cycle [50]. Previous studies have shown that CENPE was dysregulated in various malignancies, such as epithelial ovarian cancer, prostate cancer, and breast cancer, and overexpression of CENPE was associated with promoting cell cycle progression and tumor cell growth [51]. Nevertheless, the molecular mechanism and prognostic value of CENPE in CRC remain unclear. Marker of proliferation Ki-67 (MKI67) is a proliferation-related nucleus protein gene that is widely expressed in proliferating cells [52]. Previous studies have reported the abnormally high expression of MKI67 in multiple types of cancer, including CRC, and found that MKI67 could be an independent prognostic biomarker in these types of cancer [53]. However, these genes above in our study were not significantly associated with the prognosis in CRC patients. Therefore, further investigation is required to elucidate the mechanism of these genes in the carcinogenesis, progression, and treatment of CRC.

\section{Conclusion}

In conclusion, through integrated bioinformatics analysis, we identified hub genes and the association pathways involved in the initiation and progression of CRC. These findings could improve our understanding of the molecular mechanisms underlying the progression of CRC. Furthermore, the survival analysis of hub genes showed that overexpression of CDK1 and CDC20 were associated with poor survival in patients with CRC, suggesting that CDK1 and CDC20 may have potential as biomarker for CRC diagnosis, treatment, or prognosis determination. However, valuable clues provided by this study required further studies to elucidate its biological function in CRC.

\section{Abbreviations}

AURKA: Aurora kinases A; AURKB: Aurora kinases B; BP: Biological process; CC: Cellular component; CCNA2: Cyclin A2; CDC20: Cell division cycle 20 homolog; CDC6: Cell division control protein 6 homolog; CDK1: Cyclindependent kinase 1; CENPE: Centrosome-associated protein E;

CRC: Colorectal cancer; DEGs: Differentially expressed genes; GEO: Gene Expression Omnibus; GO: Gene Ontology; KEGG: Kyoto Encyclopedia of Genes and Genomes; KIF11: Kinesin family member 11; MF: Molecular 
function; MKI67: Marker of proliferation Ki-67; NCBI: National Center for Biotechnology Information; PLK1: Polo-like kinase 1; PPI: Protein-protein interaction; TCGA: The Cancer Genome Atlas

\section{Acknowledgements}

Not applicable.

\section{Authors' contributions}

$J$ and QY designed the study. $J, Y W$, and XW performed the bioinformatics analysis and interpretation of the data. YW and XW drafted the manuscript. QY agreed to be responsible for all aspects of the work to ensure that issues of accuracy or completeness of the study were properly investigated and addressed. All authors read and approved the final manuscript.

\section{Funding}

No funding was received.

\section{Availability of data and materials}

The datasets generated and/or analyzed during the current study are available in the Gene Expression Omnibus (GEO) repository (https://www. ncbi.nlm.nih.gov/geo/query/acc.cgi?acc=GSE126092).

\section{Ethics approval and consent to participate}

Not applicable.

\section{Consent for publication}

Not applicable.

\section{Competing interests}

The authors declare that they have no competing interests.

Received: 20 January 2020 Accepted: 17 February 2020

Published online: 04 March 2020

\section{References}

1. Bray F, Ferlay J, Soerjomataram I, Siegel RL, Torre LA, Jemal A. Global cancer statistics 2018: GLOBOCAN estimates of incidence and mortality worldwide for 36 cancers in 185 countries. CA Cancer J Clin. 2018;68:394-424.

2. Chen W, Zheng R, Baade PD, Zhang S, Zeng H, Bray F, et al. Cancer statistics in China, 2015. CA Cancer J Clin. 2016;66:115-32.

3. Bode AM, Dong Z, Wang H. Cancer prevention and control: alarming challenges in China. Natl Sci Rev. 2016:3:117-27.

4. Miller KD, Siegel RL, Lin CC, Mariotto AB, Kramer JL, Rowland JH, et al. Cancer treatment and survivorship statistics, 2016. CA Cancer J Clin. 2016:66:271-89

5. De Rosa M, Pace U, Rega D, Costabile V, Duraturo F, Izzo P, et al. Genetics, diagnosis and management of colorectal cancer (review). Oncol Rep. 2015;34:1087-96.

6. Zullig LL, Smith VA, Jackson GL, Danus S, Schnell M, Lindquist J, et al. Colorectal cancer statistics from the veterans affairs central cancer registry. Clin Colorectal Cancer. 2016;15:e199-204.

7. Hu Y, Gaedcke J, Emons G, Beissbarth T, Grade M, Jo P, et al. Colorectal cancer susceptibility loci as predictive markers of rectal cancer prognosis after surgery. Genes Chromosom Cancer. 2018;57:140-9.

8. Wu Z, Liu Z, Ge W, Shou J, You L, Pan H, et al. Analysis of potential genes and pathways associated with the colorectal normal mucosa-adenomacarcinoma sequence. Cancer Med. 2018;7:2555-66.

9. Chen Z, Ren R, Wan D, Wang Y, Xue X, Jiang M, et al. Hsa_circ_101555 functions as a competing endogenous RNA of miR-597-5p to promote colorectal cancer progression. Oncogene. 2019;38:6017-34.

10. Barrett T, Wilhite SE, Ledoux P, Evangelista C, Kim IF, Tomashevsky M, et al. NCBI GEO: archive for functional genomics data sets--update. Nucleic Acids Res. 2013:41(D1):D991-5.

11. Ritchie ME, Phipson B, Wu D, Hu Y, Law CW, Shi W, et al. Limma powers differential expression analyses for RNA-sequencing and microarray studies. Nucleic Acids Res. 2015;43:e47.

12. Huang W, Sherman BT, Lempicki RA. Systematic and integrative analysis of large gene lists using DAVID bioinformatics resources. Nat Protoc. 2009;4:44-57.

13. Huang DW, Sherman BT, Tan Q Kir J, Liu D, Bryant D, et al. DAVID bioinformatics resources: expanded annotation database and novel algorithms to better extract biology from large gene lists. Nucleic Acids Res. 2007:35:169-75

14. Franceschini A, Szklarczyk D, Frankild S, Kuhn M, Simonovic M, Roth A, et al. STRING v9.1: protein-protein interaction networks, with increased coverage and integration. Nucleic Acids Res. 2013;41(D1):D808-15.

15. Kohl M. Wiese S, Warscheid B. Cytoscape: software for visualization and analysis of biological networks. Methods Mol Biol. 2011;696:291-303.

16. Cerami E, Gao J, Dogrusoz U, Gross BE, Sumer SO, Aksoy BA, et al. The cBio cancer genomics portal: an open platform for exploring multidimensional cancer genomics data. Cancer Discov. 2012;2:401-4.

17. Haeussler M, Zweig AS, Tyner C, Speir ML, Rosenbloom KR, Raney BJ, et al. The UCSC genome browser database: 2019 update. Nucleic Acids Res. 2019; 47(D1):D853-8.

18. Rhodes DR, Yu J, Shanker K, Deshpande N, Varambally R, Ghosh D, et al. ONCOMINE: a cancer microarray database and integrated data-mining platform. Neoplasia. 2004;6:1-6.

19. Kuku S, Proctor I, Loddo M, Kadalayil L, KhoshZaban M. Ledermann, et al. do cell-cycle phase-specific markers predict disease grade, stage, and outcome in cervical carcinoma? Int J Gynecol Cancer. 2015;25:1066-72.

20. Habtetsion T, Ding ZC, Pi W, Li T, Lu C, Chen T, et al. Alteration of tumor metabolism by CD4+ T cells leads to TNF-a-dependent intensification of oxidative stress and tumor cell death. Cell Metab. 2018;28:228-42.

21. Bergmann $H$, Roth $S$, Pechloff $K$, Kiss EA, Kuhn S, Heikenwälder $M$, et al. Card9-dependent $I L-1 \beta$ regulates $I L-22$ production from group 3 innate lymphoid cells and promotes colitis-associated cancer. Eur J Immunol. 2017;47:1342-53.

22. Tuttle TR, Mierzwa ML, Wells SI, Fox SR, Ben-Jonathan N. The cyclic GMP/ protein kinase $\mathrm{G}$ pathway as a therapeutic target in head and neck squamous cell carcinoma. Cancer Lett. 2016:370:279-85.

23. Browning DD, Kwon IK, Wang R. CGMP-dependent protein kinases as potential targets for colon cancer prevention and treatment. Future Med Chem. 2010;2:65-80

24. Kim MP, Zhang Y, Lozano G. Mutant p53: multiple mechanisms define biologic activity in cancer. Front Oncol. 2015;5:249.

25. Cooks T, Pateras IS, Tarcic O, Solomon H, Schetter AJ, Wilder S, et al. Mutant p53 prolongs NF-kB activation and promotes chronic inflammation and inflammation-associated colorectal cancer. Cancer Cell. 2013:23:634-46.

26. Schulz-Heddergott R, Stark N, Edmunds SJ, Li J, Conradi LC, Bohnenberger $\mathrm{H}$, et al. Therapeutic ablation of gain-of-function mutant p53 in colorectal cancer inhibits Stat3-mediated tumor growth and invasion. Cancer Cell. 2018:34:298-314.

27. Mitchell A, Hasanali SL, Morera DS, Baskar R, Wang X, Khan R, et al. A chemokine/chemokine receptor signature potentially predicts clinical outcome in colorectal cancer patients. Cancer Biomark. 2019:26:291-301.

28. Ma Y, Zhao M, Zhong J, Shi L, Luo Q, Liu J, et al. Proteomic profiling of proteins associated with lymph node metastasis in colorectal cancer. J Cell Biochem. 2010;110:1512-9.

29. Cancer Genome Atlas Network. Comprehensive molecular characterization of human colon and rectal cancer. Nature. 2012;487:330-7.

30. de Sousa E, Melo F, Vermeulen L, Vermeulen L. Wnt signaling in cancer stem cell biology. Cancers (Basel). 2016;8:60.

31. Rahbari NN, Kedrin D, Incio J, Liu H, Ho WW, Nia HT, et al. Anti-VEGF therapy induces ECM remodeling and mechanical barriers to therapy in colorectal cancer liver metastases. Sci Transl Med. 2016;8:360ra135.

32. Malumbres M, Barbacid M. Cell cycle, CDKs and cancer: a changing paradigm. Nat Rev Cancer. 2009;9:153-66.

33. Ravindran Menon D, Luo Y, Arcaroli JJ, Liu S, KrishnanKutty LN, Osborne DG, et al. CDK1 interacts with Sox2 and promotes tumor initiation in human melanoma. Cancer Res. 2018;78:6561-74

34. Liu Y, Deguchi $Y$, Tian R, Wei D, Wu L, Chen W, et al. Pleiotropic effects of PPARD accelerate colorectal tumorigenesis, progression, and invasion. Cancer Res. 2019;79:954-69.

35. Wu CX, Wang XQ, Chok SH, Man K, Tsang SHY, Chan ACY, et al. Blocking CDK1/PDK1/ $\beta$-catenin signaling by CDK1 inhibitor RO3306 increased the efficacy of sorafenib treatment by targeting cancer stem cells in a preclinical model of hepatocellular carcinoma. Theranostics. 2018:8:3737-50.

36. Kuang Y, Guo W, Ling J, Xu D, Liao Y, Zhao H, et al. Iron-dependent CDK1 activity promotes lung carcinogenesis via activation of the GP130/STAT3 signaling pathway. Cell Death Dis. 2019;10:297. 
37. Xue X, Ramakrishnan SK, Weisz K, Triner D, Xie L, Attili D, et al. Iron uptake via DMT1 integrates cell cycle with JAK-STAT3 signaling to promote colorectal tumorigenesis. Cell Metab. 2016;24:447-61.

38. Bury M, Le Calvé B, Lessard F, Dal Maso T, Saliba J, Michiels C, et al. NFE2L3 controls colon cancer cell growth through regulation of DUX4, a CDK1 inhibitor. Cell Rep. 2019;29:1469-81.

39. Zhang P, Kawakami H, Liu W, Zeng X, Strebhardt K, Tao K, et al. Targeting CDK1 and MEK/ERK overcomes apoptotic resistance in BRAF-mutant human colorectal cancer. Mol Cancer Res. 2018;16:378-89.

40. Gayyed MF, El-Maqsoud NM, Tawfiek ER, El Gelany SA, Rahman MF. A comprehensive analysis of CDC20 overexpression in common malignant tumors from multiple organs: its correlation with tumor grade and stage. Tumour Biol. 2016;37:749-62.

41. Kidokoro T, Tanikawa C, Furukawa Y, Katagiri T, Nakamura Y, Matsuda K. CDC20, a potential cancer therapeutic target, is negatively regulated by p53. Oncogene. 2008:27:1562-71.

42. Wu WJ, Hu KS, Wang DS, Zeng ZL, Zhang DS, Chen DL, et al. CDC20 overexpression predicts a poor prognosis for patients with colorectal cancer. J Transl Med. 2013;11:142.

43. Hadjihannas MV, Bernkopf DB, Brückner M, Behrens J. Cell cycle control of Wnt/ $\beta$-catenin signalling by conductin/axin2 through CDC20. EMBO Rep. 2012:13:347-54

44. Pohl A, Azuma $M$, Zhang $W$, Yang $D$, Ning $Y$, Winder $T$, et al. Pharmacogenetic profiling of Aurora kinase $B$ is associated with overall survival in metastatic colorectal cancer. Pharm J. 2011;11:93-9.

45. Goos JA, Coupe VM, Diosdado B, Delis-Van Diemen PM, Karga C, Beliën JA et al. DeCoDe PET group: Aurora kinase a (AURKA) expression in colorectal cancer liver metastasis is associated with poor prognosis. Br J Cancer. 2013; 109:2445-52.

46. Zhang J, Wang Y, Shen Y, He P, Ding J, Chen Y. G9a stimulates CRC growth by inducing p53 Lys373 dimethylation-dependent activation of Plk1. Theranostics. 2018:8:2884-95.

47. Cai J, Wang H, Jiao X, Huang R, Qin Q, Zhang J, et al. The RNA-binding protein HuR confers oxaliplatin resistance of colorectal cancer by upregulating CDC6. Mol Cancer Ther. 2019:18:1243-54.

48. Imai T, Oue N, Sentani K, Sakamoto N, Uraoka N, Egi H, et al. KIF11 is required for spheroid formation by oesophageal and colorectal cancer cells. Anticancer Res. 2017;37:47-55.

49. Gan Y, Li Y, Li T, Shu G, Yin G. CCNA2 acts as a novel biomarker in regulating the growth and apoptosis of colorectal cancer. Cancer Manag Res. 2018;10:5113-24.

50. Sardar HS, Luczak VG, Lopez MM, Lister BC, Gilbert SP. Mitotic kinesin CENPE promotes microtubule plus-end elongation. Curr Biol. 2010;20:1648-53.

51. Zhu X, Luo X, Feng G, Huang $H$, He Y, Ma W, et al. CENPE expression is associated with its DNA methylation status in esophageal adenocarcinoma and independently predicts unfavorable overall survival. PLoS One. 2019;14: e0207341

52. Scholzen T, Gerdes J. The Ki-67 protein: from the known and the unknown. J Cell Physiol. 2000;182:311-22.

53. Xiong DD, Zeng CM, Jiang L, Luo DZ, Chen G. Ki-67/MKI67 as a predictive biomarker for clinical outcome in gastric cancer patients: an updated metaanalysis and systematic review involving 53 studies and 7078 patients. J Cancer. 2019;10:5339-54.

\section{Publisher's Note}

Springer Nature remains neutral with regard to jurisdictional claims in published maps and institutional affiliations.

Ready to submit your research? Choose BMC and benefit from:

- fast, convenient online submission

- thorough peer review by experienced researchers in your field

- rapid publication on acceptance

- support for research data, including large and complex data types

- gold Open Access which fosters wider collaboration and increased citations

- maximum visibility for your research: over $100 \mathrm{M}$ website views per year

At $\mathrm{BMC}$, research is always in progress.

Learn more biomedcentral.com/submissions 